\title{
Benign aqueduct stenosis in adults
}

\author{
M. J. G. HARRISON ${ }^{1}$, C. M. ROBERT, AND D. UTTLEY \\ From the National Hospital for Nervous Diseases, Queen Square, \\ and Atkinson Morley's Hospital, Wimbledon, London
}

SYNOPSIS A series of 55 cases is described in which hydrocephalus associated with non-neoplastic narrowing of the Sylvian aqueduct produced symptoms for the first time in adult life. The clinical features of the patients and their investigation are described and discussed.

Hydrocephalus may result from narrowing or obstruction of the aqueduct of Sylvius by small tumours (Petit-Dutaillis et al., 1950). The commonest neoplasm in this situation is an astrocytoma (Stookey and Scarff, 1936), but angiomata (Graf, 1946) and lipomata (Beckett et al., 1950) have been described, as well as other lesions in the region of the quadrigeminal plate cistern such as aneurysms (David et al., 1951) and arachnoid cysts (Harrison, 1971). Post-meningitis ependymitis may lead to similar aqueductal obstruction (Christensen, 1942) and other cases have been attributed to syphilis and tuberculosis (Globus and Bergman, 1946).

The aetiology of cases not due to tumours or infection is controversial (Drachman and Richardson, 1961). Russell (1949) described four types of abnormality-forking, septum formation, gliosis, and simple stenosis. She argued from the histological evidence, and from associated congenital anomalies, that the aqueduct stenosis was likely to be developmental in origin. This view has been shared by many workers (Globus and Bergman, 1946; Beckett et al., $1950)$ but also vigorously challenged. Drachman and Richardson (1961) described an important example in which forking occurred at one level of the aqueduct, and gliosis at another. They also suggested that the effects of inflammatory disease might have produced many of the changes described by Russell. Recently Williams (1973) has proposed that in many cases the aqueduct stenosis is due to passive collapse of the iter under the pressure exerted by distension

1 Address for reprints: Dr M. J. G. Harrison, Department of Neurology, The Middlesex Hospital, London W1N 8AA. of the lateral ventricles, and is therefore secondary to the hydrocephalus rather than its primary cause.

It is thus clear that a wide variety of pathological and pathophysiological changes may lead to obstruction of the aqueduct. Most examples occur in children and their recognition and surgical management are well described (Ingraham and Matson, 1954). Those cases which come to light for the first time in adult life are less common and have tended to receive less attention. It was felt therefore that it would be of interest to review the clinical features of a series of such patients. For the purposes of the study 'aqueduct stenosis' was taken to imply hydrocephalus with obstruction of the Sylvian aqueduct, due neither to neoplasms or meningitis.

\section{METHODS}

All patients over the age of 16 years at the time of their first admission to either the National Hospital, Queen Square, or to Atkinson Morley's Hospital, Wimbledon, were considered. The period covered was from 1938 to 1969. Cases were excluded if neurological abnormality dated back to infancy, or if there was a reliable history of meningitis or subarachnoid haemorrhage that might have been aetiologically responsible for the aqueduct stenosis. Similarly, if there was any doubt about the diagnosis, and in particular if a neoplastic stricture of the aqueduct was possible, the case was not included.

The diagnosis had been made radiologically in each case, and in a few patients confirmation was obtained at exploratory craniotomy (seven patients) or at postmortem examination (12 patients). The radiographic criteria employed were in keeping with those outlined by Schechter and Zingesser (1967). 
Case notes were studied retrospectively, attention being paid to the clinical presentation, and to the findings on neurological examination. The results of investigations (electroencephalography, radiography) were taken from the reports made at the time. Where radiological reports suggested some doubt about the diagnosis, the films were kindly reviewed by Dr $\mathbf{H}$. Swanson and the case excluded, if doubt still remained about the benign nature of the obstruction.

\section{RESULTS}

Fifty-five cases were collected, 28 male and 27 female. The age distribution, shown in Table 1, ranged from 16 to 62 years at the time of diagnosis.

Patients dated the onset of their symptoms from two days to 40 years before admission. A long history of 10 years or more was present in seven cases; 19 of the group $(35 \%)$ gave a history of under 12 months (Table 2).

TABLE 1

AGE AT DIAGNOSIS

\begin{tabular}{lcccccc}
\hline Age (yr) & $<20$ & $20-29$ & $30-39$ & $40-49$ & $50-59$ & $>60$ \\
Number & 7 & 20 & 10 & 10 & 7 & 1 \\
\hline
\end{tabular}

TABLE 2

DURATION OF SYMPTOMS

\begin{tabular}{lcccc}
\hline Duration (yr) & $<1$ & $1-5$ & $6-10$ & $>10$ \\
Number & 19 & 26 & 3 & 7 \\
& $(35 \%)$ & $(47 \%)$ & $(5 \%)$ & $(13 \%)$ \\
\hline
\end{tabular}

SYMPTOMS The commonest initial symptoms were headache (20 cases) visual deterioration (11), and gait disturbance (nine). Five patients presented with epilepsy (Table 3 ).

Headache, visual loss, mental deterioration, and gait disturbance were most common, when all symptoms were considered (Table 4).

INDIVIDUAL SYMPTOMS 1 Headache Those patients who had marked headache commonly described the pain as occurring in attacks. These were either brief lasting seconds or minutes, or
TABLE 3

FIRST SYMPTOM

\begin{tabular}{lrc}
\hline & No. & Per cent \\
\hline Headache & 20 & 36 \\
Visual loss & 11 & 20 \\
Gait disturbance & 9 & 16 \\
Epilepsy & 5 & 9 \\
Falls & 3 & 5 \\
Dementia & 2 & 4 \\
Vertigo & 2 & 4 \\
Miscellaneous & 3 & 5 \\
\hline
\end{tabular}

TABLE 4

ALL SYMPTOMS

\begin{tabular}{lcc}
\hline & No. & Per cent \\
\hline Headache & 32 & 58 \\
Visual loss & 22 & 40 \\
Mental deterioration & 17 & 31 \\
Gait disturbance & 16 & 29 \\
Falls & 13 & 24 \\
Endocrine & 10 & 18 \\
Nausea, vomiting & 9 & 16 \\
Epilepsy & 8 & 15 \\
Incontinence & 7 & 13 \\
Vertigo & 6 & 11 \\
Weak legs & 4 & 7 \\
Hemiparesis or & & \\
$\quad$ hemianaesthesia & 4 & 7 \\
Diplopia & 3 & 5 \\
Dysarthria & 1 & \\
Deafness & 1 & \\
\hline
\end{tabular}

continued for several days, with relief between attacks. In such attacks, nausea, vomiting, and drowsiness were common, and on occasion headache was also accompanied by a sensation of weakness in the legs or an actual fall. In other patients the headache was more continuous. The site varied, being commonly frontal, though occipital and bitemporal headache was described by individual patients. The headache usually had the characteristics of that associated with raised intracranial pressure, with exacerbations with coughing, sneezing, straining, and stooping.

2. Visual deterioration In 11 instances declining visual acuity proved to have been the initial disturbance. In six cases unilateral blurring of vision occurred and in five there was bilateral involvement. In one case bilateral temporary blurring of vision accompanied paroxysmal headache and vomiting. In all the other patients 
a progressive decline in acuity occurred. The rate varied considerably from a rapid loss of vision in one eye over the course of a week to a slowly progressive decline in acuity in both eyes over 20 years. By the time of admission for investigation 22 of the 55 patients $(40 \%)$ had visual loss of varying degree (VA <6/12). Three of the seven patients with very long histories (over 10 years) had initial visual symptoms.

3. Mental deterioration On detailed history taking, a complaint by the patient or relatives of deterioration of memory and concentration was common (17 of 55 or $31 \%$ ). The change was usually mild but in two patients the deterioration was sufficiently marked to bring the patient to medical attention in its own right.

4. Gait disturbance A difficulty with gait was reported by 16 of the 55 patients and formed the presenting feature in nine. The difficulty was usually of unsteadiness with a tendency to stagger. The onset was insidious with, for example, patients initially giving up sporting activities as their coordination became impaired. By the time of presentation many had a long history of unsteadiness on their feet. Two of the patients with a total history of over 10 years were in the group presenting with gait disturbance.

5. Falls (Falling in association with severe unsteadiness was not included in this category.) Thirteen patients described sudden falls. In many cases these were unexpected and were indistinguishable from drop attacks. Others reported a tendency to fall forwards or backwards, and yet others described a momentary loss of consciousness at the moment of falling though no other features suggested epilepsy. One in four had suffered from one of these varieties of fall.

6. Endocrine symptoms Symptoms suggestive of an endocrine abnormality occurred in $10 \%$ of the patients.

Four women reported a change in their menstrual cycles. One experienced an increase in the frequency of her periods, the others irregularity or amenorrhoea.

Three patients developed symptoms suggestive of hypothyroidism, one after thyroidectomy for thyrotoxicosis, the others without precipitating factors. In all three the time of onset of the symptoms coincided with that of the other manifestations of their aqueduct stenosis.

Two patients developed obesity, one rapidly, the other in association with an abnormal distribution of body hair in late adolescence. One other patient described a marked increase in thirst and in volume of urine produced.

7. Epilepsy Eight of the patients had had epileptic seizures by the time of admission, and in five epilepsy was the reason for referral. In two cases attacks of temporal lobe origin occurred; one had focal sensory attacks, and six had grand mal seizures. Three of the five patients presenting with epilepsy had no other symptoms; one had epilepsy and acute weight gain, and one had endocrine and visual problems. Three had no physical signs at the time of admission, highlighting the difficult diagnostic problem posed by these patients.

8. Incontinence Seven patients described disturbances of urinary sphincter control, amounting to incontinence in six and frequency and urgency in the absence of infection in one Faecal incontinence occurred in one patient. In two of the patients there was no associatedo mental impairment.

PHYSICAL SIGNS The commonest findings on examination were of mild intellectual deterioration, papilloedema or optic atrophy, reduced visual acuity, ataxia, and bilateral pyramidal tract signs.

1. Ophthalmological findings Papilloedema was the single most common finding, being present in $29(53 \%)$. In seven of these patients there was coexistent optic pallor. In another 12 patients optic atrophy was noted with no definite evidence of disc swelling. The visual acuity was reduced below 6/12 in one or both eyes in 17 instances (approximately $30 \%$ ). No abnormality of the visual fields was recorded in 43 patients. In two patients the blind spots were considerably enlarged, and in three peripheral fields restricted (in association with severe papilloedema). Three patients had scotomata. In three others quadrantic or hemianopic defects were encountered. 
One patient had a nasal hemianopia in one eye and a general peripheral restriction in the other. Another had a temporal hemianopia in one eye. Thus five patients had field defects suggestive of lesions in the optic nerves or chiasm.

2. Intellectual changes In 20 patients $(36 \%)$ there was some evidence of intellectual deterioration. The dementia was in most cases mild and had occasioned few complaints. Mild memory loss and impaired learning were detected on testing, however. The frequency of this finding may well be much higher, as not all patients were tested formally.

3. Ataxia In 16 patients the gait was ataxic. In six of these patients testing also revealed limb ataxia. In another six ataxia of limbs was present but the gait was felt to be normal.

4. 'Pyramidal' signs A mild hemiparesis was detected in 10 patients, a paraparesis in two. Tendon reflexes were increased in 12 cases. The plantar responses were extensor in 11 (bilateral in six). In all, 24 showed 'pyramidal tract' signs. A further four patients had hemisensory findings.

\section{Brain-stem signs Nystagmus was recorded} in four subjects. Two patients had sixth nerve palsies, and one had sensory loss and weakness in the fifth nerve territory on one side. One patient showed features of a partial Parinaud syndrome with defective upward gaze and convergence, with very sluggishly reacting pupils (Swash, 1974). One other had unreacting pupils.

6. Miscellaneous Five patients had anosmia (bilateral in two), which was thought to be related to the hydrocephalus. One was hypothyroid clinically. Four patients had some evidence of enlargement of the skull. In three cases there was no abnormality on examination.

INVESTIGATIONS Electroencephalography Electroencephalographic reports were available for 32 patients taken at the time of their initial admission for diagnosis. Ten of these had been reported as normal. In four instances epileptic features-that is, spikes-were recorded. In five nonspecific abnormalities were reported-for example, bitemporal excess of low voltage theta activity. One patient had slowing of the alpha rhythm in association with hypothyroidism.

The commonest abnormality, which occurred in 13 cases, was the presence of intermittent bilateral synchronous episodes or bursts of slow wave activity, usually in the delta range. These were usually bifrontal in distribution and of medium or high voltage. They were usually reported as indicating a disturbance of midline structures and are identical with the changes in other conditions in which brain-stem structures are affected by lesions causing increased intracranial pressure.

Radiology Plain skull films had been taken in 53 cases. Six were reported on as normal. Fortytwo $(76 \%)$ were thought to show the effects of 'chronic raised intracranial pressure'. In three cases the skull vault appeared larger than normal, and in one the small size of the posterior fossa was noted. An abnormality of the pituitary fossa was noted in 25 instances. In two patients the fossa appeared long and shallow, in the other 23 $(42 \%)$ the sella turcica was 'enlarged'.

The characteristic abnormality of the sella turcica in benign stenosis of the aqueduct has recently been described by $\mathrm{Du}$ Boulay and Trickey (1970). The sella shape is abnormal with a short dorsum sellae, and a long steep anterior wall. This abnormality was probably at times what was being described in previous papers (and in early cases in this series), as 'enlargement of the sella'. Du Boulay and Trickey found that the non-specific effects of raised intracranial pressure were never the sole abnormalities in the skull films of patients with aqueduct stenosis.

Angiography Carotid angiography had been carried out in 13 patients. In each case, there was evidence of enlargement of the lateral ventricle. No other abnormality was reported.

Vertebral angiograms had been performed in three cases. Two were reported as normal. In one patient the basilar artery was noted to be flattened against the clivus.

Pneumoencephalography Sixteen patients had lumbar pneumoencephalograms; air failed to enter the ventricular system in six instances. In 10 cases air outlined a fourth ventricle of normal size and configuration. There was no evidence of 
lateral shift of the fourth ventricle in any case, and no evidence of a mass lesion. In one patient a small amount of air passed up a narrowed aqueduct.

Ventriculography Twenty-eight patients were studied by pneumoventriculography alone, 10 with iodophendylate (Myodil) ventriculography alone, and in 15 both air and Myodil were used. In every case symmetrical enlargement of the lateral and 3rd ventricles was demonstrated. The posterior end of the 3rd ventricle was outlined in each case and the aditus of the aqueduct visualized. Varying abnormalities were reported from a blunt obstruction to a tapered stricture. The wall of the upper aqueduct showed no irregularity, and the aqueduct was not displaced from the midline. In one case a small amount of Myodil finally passed down a narrowed aqueduct.

\section{DISCUSSION}

This study as well as previous smaller series, demonstrates that the clinical manifestations of benign aqueductal stenosis not infrequently arise for the first time in adult life (Paine and McKissock, 1955; Friedmann, 1964; Wilkinson et al., 1966). The length of the history is often very striking, a measure of the mild nature of the symptoms in many patients. Some cases have been described in which hydrocephalus associated with aqueduct stenosis had occasioned few if any symptoms, and was an unexpected finding at necropsy (McHugh, 1964). Some patients who gave a short history had evidence that their hydrocephalus had been longstanding. Thus one patient who became confused and ataxic after a fall had optic atrophy, and radiographs of the skull showed marked erosion of the dorsum sellae. Sudden changes in cerebrospinal fluid hydrodynamics may cause a breakdown in borderline compensation of partial obstruction (Shelden et al., 1930), and so precipitate clinical deterioration. Very short histories of raised intracranial pressure and of sudden death are both described (McHugh, 1964).

Our study adds no new information about the aetiology of this condition. There was no evidence of a preceding illness. Experimental studies have demonstrated that gliosis of the aqueduct may follow mumps infection in the suckling hamster, in the absence of any overt acute infection (Johnson et al., 1967). While a similar neonatal infection may be involved in human cases, no evidence has been produced in favour of this hypothesis.

It remains possible that some of our cases are harbouring undetected neoplasms. The radiological distinction may be impossible. Schechter and Zingesser (1967) found one case with an apparently non-neoplastic aqueduct stenosis which had, at necropsy, a small astrocytoma blocking the aqueduct. The rigid criteria adopted, the negative explorations, and the length of the follow-up make it unlikely that a significant number of our cases are due to such causes.

The study of the symptoms revealed that headache, visual failure, mental dulling, and episodic ataxia or falls form a characteristic complex. The headache often had the features of intermitten raised intracranial pressure (Kelly, 1951). Nausea and vomiting often accompanied bouts of head ache and in a few cases loss of awareness op coma followed (Wilkinson et al., 1966).

The prevalence of visual failure was striking and has received insufficient attention in the past. Asymmetrical reduction in visual acuits $\stackrel{\Phi}{\varnothing}$ was frequently encountered and found to be associated with optic disc pallor.

Blurring of the optic discs was also common. The optic atrophy did not always have the appearances of consecutive atrophy however, and the occasional occurrence of visual field defects (Lassman et al., 1960) and the abnormalities of the pituitary fossa on plain radiographs suggest that direct optic nerve or chiasmatic compression occurs from the expanded anterior end of the hydrocephalic third ventricle (Sheldon et al., 1930). Hypothalamic and pituitary damage from a similar cause presumably accounts for the occasional endocrine symptoms (Rhein, 1925). Previous authors have noted the occasional occurrence of obesity, gynaecomastia, amenorrhoea, hypopituitarism (Sheldon et al., 1930), and polydipsia and polyuria (Strauch, 1919). The frequency of such symptoms in this series is similar to that noted by Paine and McKissock (1955). Anosmia may also result $\frac{7}{0}$ from hydrocephalic distension of the anterior end of the third ventricle with pressure on the $\widetilde{N}$ olfactory bulb (Rhein, 1925). 
Drop attacks or unexpected staggering or falls were common. These are thought to be due to sudden changes in pressure relationships as may occur with colloid cysts of the third ventricle (Kelly, 1951).

The cerebellar signs are more difficult to understand. Compression within the posterior fossa has been suggested (Jakubowski and Jefferson, 1972), but there has been little pathological information on which to decide this point.

Mental changes were common on inquiry, and testing revealed slight impairment of memory in almost half the group (Friedmann, 1964). The changes were obtrusive in only a few, but these are important cases as aqueduct stenosis must be included in the differential diagnosis of dementia.

Bilateral pyramidal tract signs and even spastic paraparesis may be seen in these patients as in others with hydrocephalus (McHugh, 1964). The involvement of pyramidal tract fibres has usually been attributed to stretching around dilated ventricles (Yakovlev, 1947), but there has been no direct pathological confirmation for this.

Epilepsy occurred in $15 \%$ of the group. Greitz et al. (1971) recently reported a similar prevalence $(18.5 \%)$. In both these series grand mal fits predominated but focal seizures also occurred. It is of clinical importance that in some instances there were no other symptoms and no abnormality on full neurological examination.

In the investigation of these cases plain skull radiographs provide information of diagnostic value in the great majority ( $\mathrm{Du}$ Boulay and Trickey, 1970). The findings on ventriculography are well described in the detailed study of Schechter and Zingesser (1967) and the present series adds nothing to their account.

The surgical management of these cases will be discussed elsewhere (Uttley et al., in preparation).

We are grateful to the physicians and surgeons of the National Hospital, Queen Square, and of Atkinson Morley's Hospital, Wimbledon, for permission to study patients under their care.

\section{REFERENCES}

Beckett, R. S., Netsky, M. G., and Zimmerman, H. M. (1950). Developmental stenosis of the aqueduct of Sylvius. American Journal of Pathology, 26, 755-787.
Christensen, E. (1942). Four cases of chronic granular ependymitis. Acta Psychiatrica et Neurologica, 17, 123-138.

David, M., Morice, J., and Adam (1951). Sténose de l'aqueduc de Sylvius par anévrysme des artères cérébelleuses supérieures. Revue Neurologique, 84, 313-314.

Drachman, D. A., and Richardson, E. P., Jr (1961). Aqueductal narrowing, congenital and acquired. Archives of Neurology (Chic.), 5, 552-559.

Du Boulay, G., and Trickey, S. (1970). The sella in aqueduct stenosis and communicating hydrocephalus. British Journal of Radiology, 43, 319-326.

Friedmann, G. (1964). Die nicht durch Tumor bedingte Aquäduktstenose und ihr Nachweis im Röntgenübersichtsbild. Der Radiologe, 4, 190-194.

Globus, J. H., and Bergman, P. (1946). Atresia and stenosis of the aqueduct of Sylvius. Journal of Neuropathology and Experimental Neurology, 5, 342-363.

Graf, C. (1946). Angiomatous malformation of the Sylvian aqueduct with remarks on management of aqueductal obstructions. Journal of Neuropathology and Experimental Neurology, 5, 43-53.

Greitz, T., Levander, B. E., and López, J. (1971). High blood pressure and epilepsy in hydrocephalus due to stenosis of the aqueduct of Sylvius. Acta Neurochirurgica, 24, 201-206.

Harrison, M. J. G. (1971). Cerebral arachnoid cysts in children. Journal of Neurology, Neurosurgery, and Psychiatry, 34, 316-323.

Ingraham, F. D., and Matson, D. D. (1954). Neurosurgery of Infancy and Childhood. Thomas: Springfield, Ill.

Johnson, R. T., Johnson, K. P., and Edmonds, C. J. (1967). Virus-induced hydrocephalus: development of aqueductal stenosis in hamsters after mumps infection. Science, 157, 1066-1067.

Jakubowski, J., and Jefferson, A. (1972). Axial enlargement of the 3rd ventricle, and displacement of the brain-stem in benign aqueduct stenosis. Journal of Neurology, Neurosurgery, and Psychiatry, 35, 114-123.

Kelly, R. (1951). Colloid cysts of the third ventricle. Analysis of twenty-nine cases. Brain, 74, 23-65.

Lassman, L. P., Cullen, J. F., and Howat, J. M. L. (1960). Stenosis of the aqueduct of Sylvius. American Journal of Ophthalmology, 49, 261-266.

McHugh, P. R. (1964). Occult hydrocephalus. Quarterly Journal of Medicine, 33, 297-308.

Paine, K. W. E., and McKissock, W. (1955). Aqueduct stenosis: clinical aspects, and results of treatment by ventriculocisternostomy (Torkildsen's operation). Journal of Neurosurgery, 12, 127-145.

Pennybacker, J. (1940). Stenosis of the aqueduct of Sylvius. Proceedings of the Royal Society of Medicine, 33, 507-512.

Petit-Dutaillis, D., Thiébaut, F., Berdet, H., and Barbizet, J. (1950). A propos des sténoses de l'aqueduc de Sylvius d'origine non tumorale de l'adolescent et de l'adulte. Revue Neurologique, 82, 417-421.

Rhein, J. H. W. (1925). Hypophysial pressure symptoms due to hydrocephalus causing cystlike distension of the third ventricle. Archives of Neurology and Psychiatry (Chic.), 13, 71-79.

Russell, D. S. (1949). Observations on the Pathology of Hydrocephalus. Medical Research Council Special Report Series No. 265. H.M.S.O.: London.

Schechter, M. M., and Zingesser, L. H. (1967). The radiology of aqueductal stenosis. Radiology, 88, 905-916.

Shelden, W. D., Parker, H. L., and Kernohan, J. W. (1930). Occlusion of the aqueduct of Sylvius. Archives of Neurology and Psychiatry (Chic.), 23, 1183-1202. 
Stookey, B., and Scarff, J. E. (1936). Occlusion of the aqueduct of Sylvius by neoplastic and non-neoplastic processes with a rational surgical treatment for relief of resultant obstructive hydrocephalus. Bulletin of the Neurological Institute of New York, 5, 348-377.

Strauch, A. (1919). Hypophysial dystrophy in hydrocephalus. Journal of the American Medical Association, 72, 1731-1734.

Swash, M. (1974). Periaqueductal dysfunction (the Sylvian aqueduct syndrome): a sign of hydrocephalus? Journal of Neurology, Neurosurgery, and Psychiatry, 37, 21-26.
Uttley, D., Robert, C. M., and Harrison, M. J. G. (1975). In preparation.

Wilkinson, H. A., LeMay, M., and Drew, J. H. (1966). Adult aqueductal stenosis. Archives of Neurology, 15, 643648.

Williams, B. (1973). Is aqueduct stenosis a result of hydrocephalus? Brain, 96, 399-412.

Yakovlev, P. I. (1947). Paraplegias of hydrocephalics. American Journal of Mental Deficiency, 51, 561-576. 